\title{
Acute Ileal Perforation Caused by Radiation Enteritis After Restoration
}

\author{
Jong Beom Kim¹, Jong Lyul Lee ${ }^{1}$, Seong Ho Park², Jihun Kim, Jin Cheon Kim \\ ${ }^{1}$ Division of Colon and Rectal Surgery, Department of Surgery, ${ }^{2}$ Department of Radiology, and ${ }^{3}$ Department of Pathology, Asan Medical \\ Center, University of Ulsan College of Medicine, Seoul, Korea
}

Few radiation-induced bowel perforations have been reported to date. Furthermore, perforation after ileal restoration in asymptomatic patients is rare. We report the case of a 61 -year-old man who was administered preoperative chemoradiotherapy for advanced rectal cancer. The patient underwent ultra-low anterior resection with ileal diversion, followed by ileal restoration. Perforation was detected 9 days after restoration, and he underwent a right hemicolectomy. The histologic evaluation indicated ileal perforation caused by acute radiation enteritis.

Keywords: Rectal neoplasms; Chemoradiotherapy; Radiation; Enteritis

\section{INTRODUCTION}

In recent years, preoperative chemoradiotherapy (CRT) combined with total mesorectal excision has become a well-established approach for the reduction of local recurrence following the treatment of advanced rectal cancer [1]. As many rectal cancer patients undergo preoperative CRT, the complications associated with radiation should not be overlooked. Gastrointestinal symptoms following radiotherapy can be caused by intestinal strictures, intestinal fistula, de novo or recurrent neoplasia, small bowel bacterial overgrowth, bile salt malabsorption, fat or carbohydrate malabsorption, inflammatory bowel disease, pancreatic insufficiency, or irritable bowel syndrome, presenting with variable degrees [2]. In addition, rare cases of perforation, which can lead to generalized peritonitis and sometimes mortality, have been reported [3].

Herein, we report a rare complication of radiation enteritis, namely ileal perforation, after ileal restoration in a patient who underwent preoperative CRT.

Received: May 29, 2020 - Revised: Jul 19, 2020 - Accepted: Jul 30, 2020 Correspondence to: Jin Cheon Kim, M.D.

Department of Surgery and Institute of Innovative Cancer Research, Asan Medical Center, University of Ulsan College of Medicine, 88 Olympic-ro 43-gil, Songpa-gu, Seoul 05505, Korea

Tel: +82-2-3010-3489, Fax: +82-2-474-9027

E-mail: jckim@amc.seoul.kr

ORCID: https://orcid.org/0000-0003-4823-8619

(C) 2021 The Korean Society of Coloproctology

This is an open-access article distributed under the terms of the Creative Commons Attribution NonCommercial License (https://creativecommons.org/licenses/by-nc/4.0) which permits unrestricted noncommercial use, distribution, and reproduction in any medium, provided the original work is properly cited.

\section{CASE REPORT}

This study was reviewed and approved by the Institutional Review Board of the Asan Medical Center in Seoul, Korea (No. 2020-0846). Written informed consent was obtained for publication of this case report and accompanying images.

A 61-year-old man visited the hospital with pathologically confirmed rectal cancer $3 \mathrm{~cm}$ from the anal margin, identified using colonoscopy. Magnetic resonance imaging (MRI) scan revealed a tumor with perirectal fat infiltration. Preoperative CRT with capecitabine was administered at a cumulative dose of 5,040 cGY, divided into 28 cycles. Following preoperative CRT, sigmoidoscopy revealed the mass size to have decreased. MRI scan also revealed a decrease in the mass size, and the residual lesion contained more fibrous than tumor tissue. In addition, contrast-enhanced computed tomography (CT) scan showed diffuse wall thickening in a distal ileal segment located in the pelvis, indicating probable radiation enteritis (Fig. 1A). Eight weeks after the completion of preoperative CRT, the patient underwent robot-assisted ultra-low anterior resection with ileal diversion. The patient recovered uneventfully and was discharged 7 days after the surgery. Postoperative histologic staging was ypT3N0, and 6 cycles of adjuvant chemotherapy with capecitabine were administered thereafter.

The patient was admitted again for ileal restoration 6 months after the operation. A small incision for ileal restoration did not permit accurate evaluation of the stricture and wall thickening or perfusion along the entire ileum. Functional end-to-end anastomosis was performed using a linear cutting stapler. Two days after the surgery, the patient's progress was unremarkable, other than 

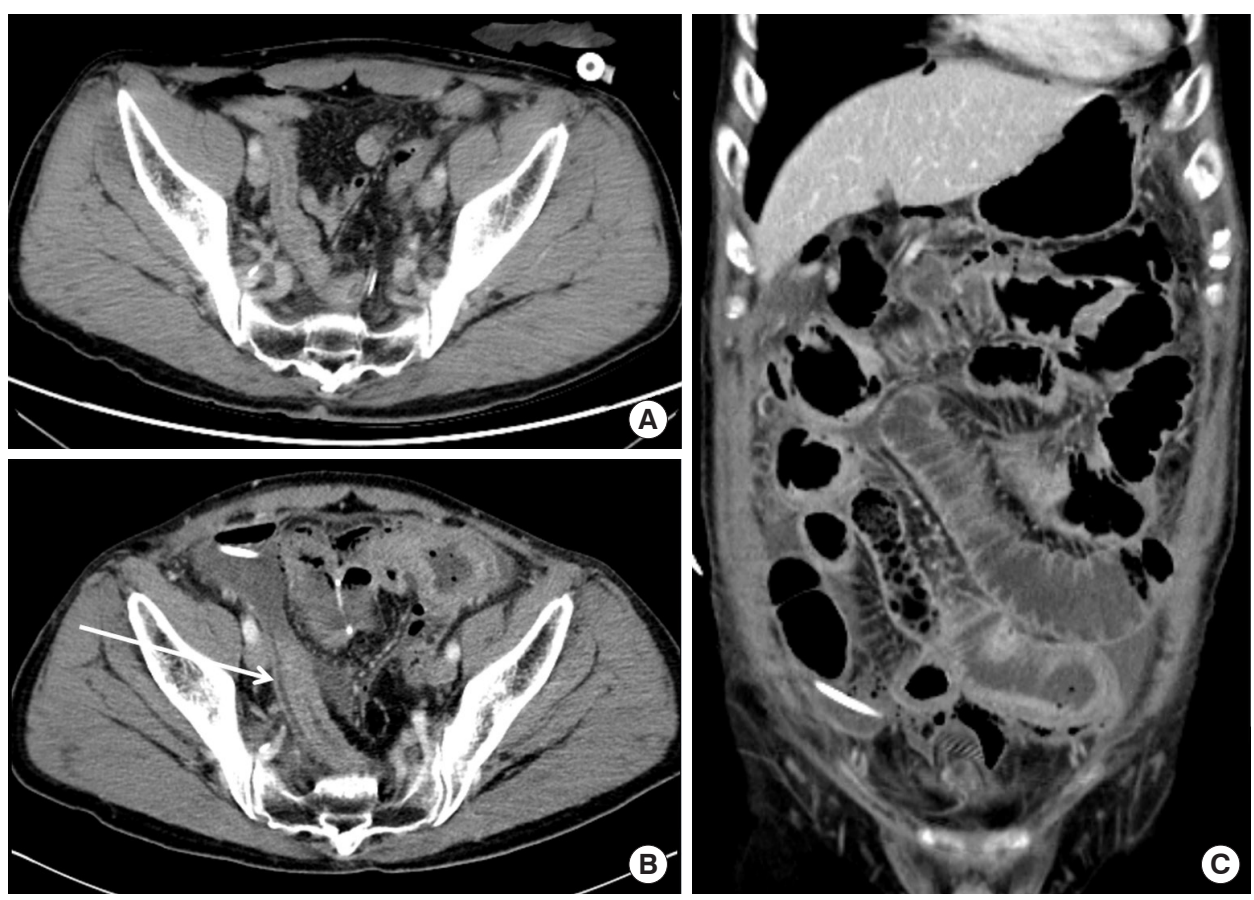

Fig. 1. Abdominal computed tomography scans. (A) Diffuse wall thickening in the distal ileal segment located in the pelvis 8 weeks after the preoperative chemoradiotherapy. (B) Small bowel obstruction following a persistent distal ileal stricture (white arrow). (C) Dilated proximal small bowel.

several episodes of diarrhea. However, 5 days after the surgery, a sudden episode of fever $\left(38.7^{\circ} \mathrm{C}\right)$ developed, along with abdominal distension and mild tenderness. CT images suggested segmental diffuse edema in the terminal ileum, resulting in small bowel stricture and some uncomplicated reactive ascites. Although the patient was treated conservatively with fasting and antibiotics, the fever did not subside, and the abdominal distension and tenderness worsened. Percutaneous catheter drainage (PCD) was performed 8 days after the surgery; $550 \mathrm{~mL}$ of fluid was drained, which was clear and serous. After drainage, the patient's distension and tenderness showed some improvement. However, 9 days after surgery, the patient exhibited aggravated symptoms, and the PCD appeared green in color and turbid in nature. In addition, an erect simple abdominal radiograph confirmed the presence of free air. CT images suggested peritonitis associated with bowel perforation, including free air, ascites, peritoneal infiltration, and thickening with enhancement (Fig. 1B, C). There was also a persistence of the previously noted diffuse segmental mural thickening in the distal ileum (i.e., presumed radiation enteritis), causing small bowel stricture with proximal dilatation (Fig. 1B, C).

Considering the CT findings and the nature of the drainage fluid, emergency surgery was planned. Surgical findings showed that the previous anastomosis was intact, and a pinpoint perforation was confirmed on the sidewall of the anastomosis (Fig. 2A). As the perforation site was identified at some distance from the PCD, the possibility of iatrogenic perforation following PCD in- sertion was less likely. A stricture, caused by radiation enteritis, was detected $10 \mathrm{~cm}$ in length in the distal bowel segment $5 \mathrm{~cm}$ above the crotch of restoration anastomosis. A limited right hemicolectomy with ileal resection (length, $42 \mathrm{~cm}$ ), including the previous stomal restoration site, was subsequently performed.

Postoperative histology revealed the perforation site with a transmural tissue defect and acute fibrinous inflammation (Fig. 2B). In addition, the affected mucosa showed marked crypt dissipation, stromal hyalinization, and telangiectasia (Fig. 2C, D). Taken together, these pathologic features are consistent with perforated severe radiation enteritis. Subsequent culture of the ascites fluid confirmed enteric aerobic and anaerobic microbes; however, the clostridium difficile toxin assay was negative. The patient was discharged without the occurrence of any subsequent events other than a few episodes of diarrhea 11 days after surgery.

\section{DISCUSSION}

Gastrointestinal adverse events following radiation therapy can occur when gastrointestinal structures are included in the radiation field. The incidence and severity of radiation enteritis depend on the dose of radiotherapy. Additional aggravating factors include age, presence of comorbidities, prior operation, tobacco abuse, and concurrent chemotherapy [3]. Enteral epithelium is affected by chemotherapy as well as radiation because of its highly proliferative potential [4]. Mucosal atrophy with atypical hyper- 


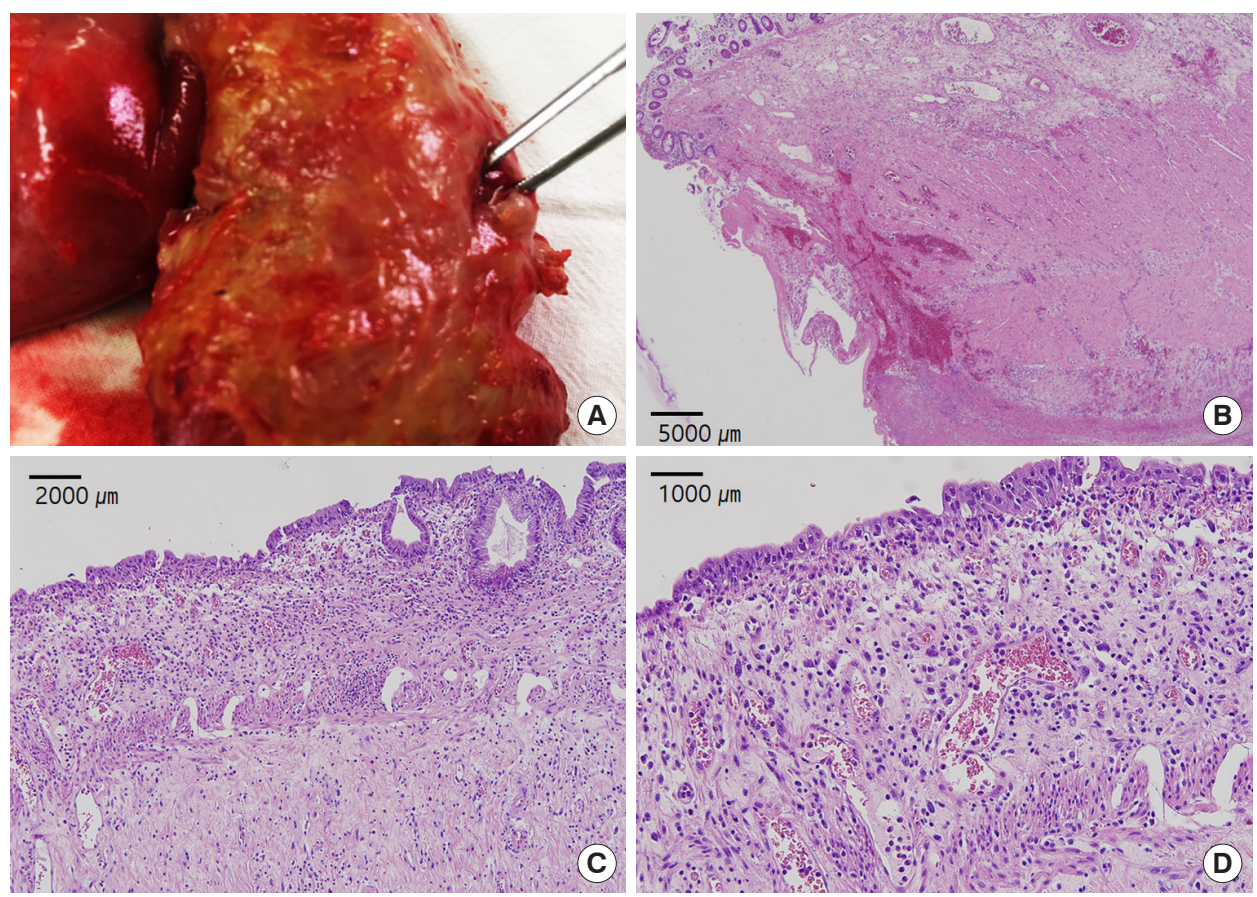

Fig. 2. Gross and histologic findings. (A) Pinpoint perforation of the sidewall of the anastomosis. (B) Perforation with acute fibrinous inflammation of the left side of the bowel wall $(\mathrm{H} \& \mathrm{E}, \times 40)$. (C) Marked crypt dropout in the mucosa (H\&E, $\times 100)$. (D) Stromal hyalinization and telangiectasia at high magnification $(\mathrm{H} \& \mathrm{E}, \times 200)$.

plastic glands and intestinal wall fibrosis, and telangiectasias can be typical histologic changes [5]. Otherwise, mucosal ulceration can also occur, sometimes developing abscess, fistula, and perforation. Fibrosis occurs as part of the healing process of the ulcer, which unfortunately produces narrowing of the enteral lumen causing stricture and even obstruction [5]. The patient did not have any comorbidities and history of surgery except for rectal cancer operation. He had a 30-pack-year smoking history, but quit smoking approximately a year before the rectal cancer diagnosis. Considering the pathogenesis of the patient, stricture caused by radiation enteritis led to obstruction and small bowel bacterial overgrowth caused by stasis, leading to stercoral enteritis and perforation caused by high pressure.

In general, the complication of radiation can be divided into acute and late, depending on the timing. Late complication is less frequent than acute complication, but it can occur in up to $40 \%$ of patients receiving radiation in the abdominopelvic area three to 6 years after radiation [3]. Complications can be graded from 0 to 5 according to the Radiation Therapy Oncology Group morbidity grading scale [3]. Depending on the severity, there are various clinical features such as enteritis, bleeding, narrowing, perforation, fistula, etc. Enteral perforation is a very uncommon complication compared with bleeding and obstruction [6].

Diagnosis should be based on a combination of the patient's clinical symptoms, history, laboratory result, imaging finding, and physical examination. The possibility of recurrent neoplasia should not be ruled out during diagnostic workup [7]. Management of chronic radiation enteritis is primarily focused on symptomatic improvement. Additionally, nutritional support should not be omitted. Symptomatic treatments include antidiarrheal agents, antiinflammatories, probiotics and antibiotics, cholestyramine, pentoxifylline and tocopherol, and hyperbaric oxygen, based on the individual status [2]. If the response to medical treatment does not meet the expectations, endoscopic therapy, including coagulation therapy and balloon dilatation, and further surgery needs to be considered [2].

This report describes a rare case of ileal perforation caused by intractable radiation enteritis after ileal restoration. In conclusion, when radiation enteritis continues to be intractable, concurrent with relevant physical, laboratory, and abdominopelvic CT findings, timely surgical intervention needs to be considered, or an irradiated bowel resection should be performed during ileal restoration.

\section{CONFLICT OF INTEREST}

No potential conflict of interest relevant to this article was reported.

\section{REFERENCES}

1. van Gijn W, Marijnen CA, Nagtegaal ID, Kranenbarg EM, Putter $\mathrm{H}$, Wiggers T, et al. Preoperative radiotherapy combined with to- 


\section{Coloproctology Jong Beom Kim, et al.}

tal mesorectal excision for resectable rectal cancer: 12-year follow-up of the multicentre, randomised controlled TME trial. Lancet Oncol 2011;12:575-82.

2. Theis VS, Sripadam R, Ramani V, Lal S. Chronic radiation enteritis. Clin Oncol (R Coll Radiol) 2010;22:70-83.

3. Turina M, Mulhall AM, Mahid SS, Yashar C, Galandiuk S. Frequency and surgical management of chronic complications related to pelvic radiation. Arch Surg 2008;143:46-52.

4. Bismar MM, Sinicrope FA. Radiation enteritis. Curr Gastroenter- ol Rep 2002;4:361-5.

5. Berthrong M, Fajardo LF. Radiation injury in surgical pathology. Part II. Alimentary tract. Am J Surg Pathol 1981;5:153-78.

6. Kavanagh BD, Pan CC, Dawson LA, Das SK, Li XA, Ten Haken RK, et al. Radiation dose-volume effects in the stomach and small bowel. Int J Radiat Oncol Biol Phys 2010;76(3 Suppl):S101-7.

7. Harb AH, Abou Fadel C, Sharara AI. Radiation enteritis. Curr Gastroenterol Rep 2014;16:383. 